\title{
A 3-channel ECG measuring system for wireless applications
}

\author{
R. Dorn ${ }^{1}$, M. Völker ${ }^{1}$, H. Neubauer ${ }^{1}$, J. Hauer ${ }^{1}$, J. Johansson ${ }^{2}$ \\ ${ }^{1}$ Fraunhofer Institute for Integrated Circuits IIS, Am Wolfsmantel 33, 91058 Erlangen, Germany \\ E-mail: robert.dorn@iis.fraunhofer.de. \\ ${ }^{2}$ EISLAB, Luleå University of Technology, 97187 Luleå, Sweden
}

\begin{abstract}
This paper describes the design and impementation of an integrated frontend for electrocardiographic (ECG) systems, realized in a $0.35 \mu \mathrm{m} 2 \mathrm{P} 4 \mathrm{M}$ CMOS process. The performance is optimized to adhere to the standard IEC60601-2-47, which defines the requirements for safety and essential performance of ambulatory ECG equipment. The system consists of three channels to measure the 3 leads of a Goldberger ECG monitoring scheme, therefore a single ended design structure was chosen to minimize the power consumption. A fourth channel is included for additional measurements. Each of the four channels contains a low power multi-bit sigma-delta modulator and a low power digital filter. Three channels are equipped with a low noise preamplifier. The supply voltage can be varied from 2.4 Volt up to 3.6 Volt. With a total power consumption of less than $2 \mathrm{~mW}$ the circuit is designed for battery operated equipment.
\end{abstract}

Keywords - ecg, low power, filter, sigma-delta.

\section{INTRODUCTION}

The field of on-line, out-of-hospital health care and health supervision is constantly expanding. Shorter or no hospital time for patients could yield large economical savings, hence the area receives attention both in medical and technical conferences and journals. Advances in technology and miniaturization of electronics have increased the number of portable, battery-operated equipment available. This is also the case for electrocardiographic (ECG) systems, making them available not only for clinical use but also for monitoring in sports or other activities.

This type of ambulatory ECG equipment is defined in the standard IEC60601-2-47 [1], which among others sets the performance requirements on this equipment. The required bandwidth of the ECG sensor is low. At the same time, the maximal signal amplitude is $6 \mathrm{mV}$ with an offset voltage up to $\pm 300 \mathrm{mV}$. For this reason a high dynamic range is required for this system. Additionally, power consumption is an important constraint for battery operated equipment.

Together with a microcontroller/bluetooth-board (MULLEModule) [2] a wireless demonstrator was realized. The embedded-webserver of the MCU-board allows to transmit the measured data to a bluetooth compatible PDA or via mobile phone to the internet.

This paper describes the system integration of a sigma-delta $(\Sigma \Delta)$ modulator together with a preamplifier and a digital fil- ter. The design of these parts is explained in section II. The layout is presented in the third section and finally measurement results are shown and discussed.

\section{SYSTEM DESIGN}

The ECG IC is designed for ambulatory recordings, like 24-hour ECG or wireless monitoring. The block diagram is shown in Fig. 1. The ASIC includes three channels that are equipped with a low noise, low power preamplifier to record a three lead Goldberger ECG. A fourth channel is included for additional measurements. All of them are realized in a single ended structure to keep the power consumption low. The output of the digitally filtered data is done serially.

The IEC60601-2-47 standard claims the following specifications for the system design:

- Noise: Input referred noise should not exceed $50 \mu \mathrm{V}_{\mathrm{pp}}$.

- Frequency response: A bandpass characteristic with passband between $0.67 \mathrm{~Hz}$ and $40 \mathrm{~Hz}$ is needed.

- Resolution: The measurement of a $10 \mathrm{~Hz}, 50 \mu \mathrm{V}_{\mathrm{pp}}$ signal should be possible.

- Input dynamic range: A $6 \mathrm{mV}_{\mathrm{pp}}$ signal shall be measured with offsets up to $\pm 300 \mathrm{mV}$.

- Input resistance: The input resistance should be higher than $10 \mathrm{M} \Omega$.

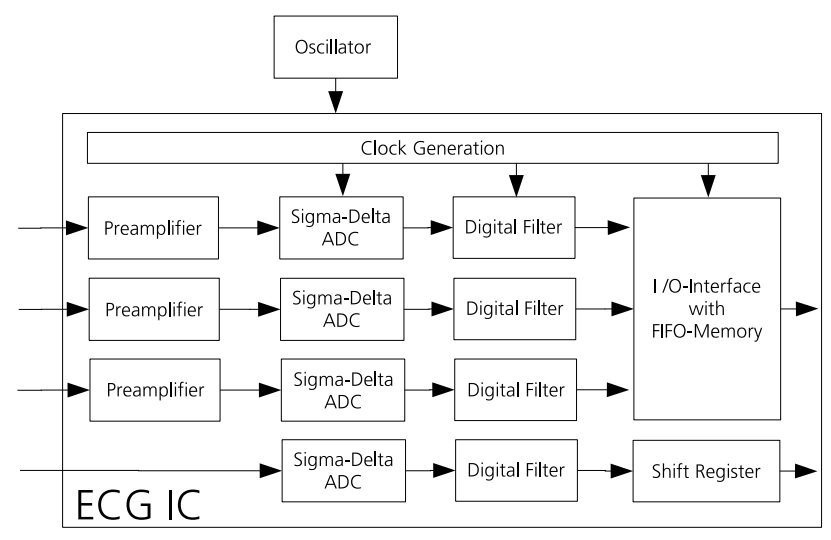

Fig. 1. System block diagram 
The main focus of the design is to achieve low power consumption. The design target is to consume less than $1 \mathrm{~mW}$ per channel.

\section{A. Preamplifier}

The preamplifier design is focused on low power consumption and low noise. In order not to affect the dynamic range of the $\Sigma \Delta$-modulator a noise level below the quantization noise of the modulator has to be reached. The equivalent noise voltage of the modulator is $7.2 \mu \mathrm{V}_{\text {rms }}$ in the baseband. Furthermore an input resistance of more than $10 \mathrm{M} \Omega$ is claimed by the IEC standard. The target preamplification of 3.5 is a tradeoff between the required resolution and offset voltages up to $\pm 300 \mathrm{mV}$. A basic two stage amplifier with resistive feedback has been chosen to fullfill this requirements. The power consumption for one amplifier is less than $200 \mu \mathrm{W}$ and the effective noise voltage is less than $7 \mu \mathrm{V}_{\text {rms }}$ up to $6 \mathrm{kHz}$.

\section{B. $\Sigma \Delta$-Modulator}

Main part in the ECG system is the analog-to-digital conversion, which is decisive for the performance of the equipment. The combination of high resolution, low bandwidth, and low power consumption is well suited to be implemented in a $\Sigma \Delta$-modulator.

The AD-converter is implemented as a first-order, 3-bit sigma-delta modulator with an oversampling ratio of 512. A test chip had shown that the single ended design achieves a dynamic range of 15 bits for signal offsets up to $\pm 1.2 \mathrm{~V}$ [3]. The measured power consumption for one single modulator is $60 \mu \mathrm{W}$ for a supply voltage of $2.4 \mathrm{~V}$. A system level schematic of the Sigma-Delta Modulator is shown in Fig. 2.

The integrator with multibit feedback is built around a single ended operational transconductance amplifier (OTA). The digital analog converter (DAC) in the feedback loop is implemented by splitting up the sampling capacitance in 7 equal $600 \mathrm{fF}$ units, Cs1 through Cs7 . Each of these consist of four symmetrically arranged $150 \mathrm{fF}$ capacitors.

Power consumption is a key factor in the choice of topology for the A/D converter. A charge transfer comparator (CTC) has been chosen, because the circuit consumes no static power, and uses very low power consumption for low frequencies.

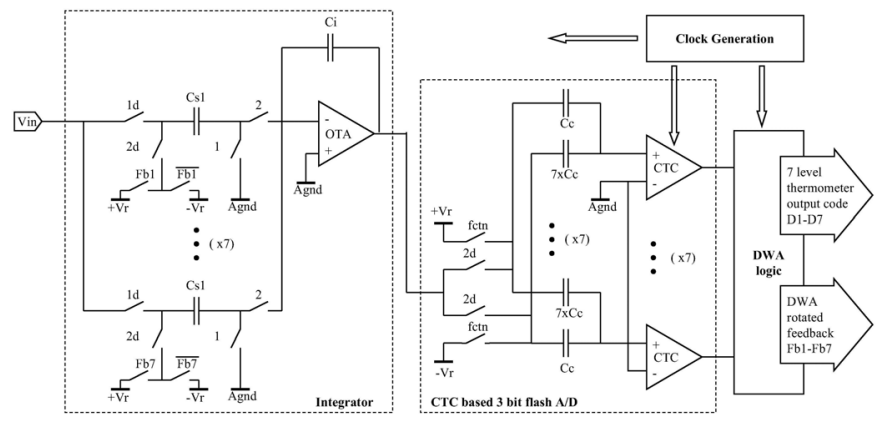

Fig. 2. Sigma-Delta Modulator
Multibit $\Sigma \Delta$-converters are sensitive to non-idealities such as mismatch in the feedback D/A converter, as these errors are added directly to the input signal and are thus not noise shaped. For target resolutions exceeding the device matching of a CMOS technology this problem must be addressed. One technique to solve this problem is to use data-weighted averaging (DWA) [3]. The implementation chosen here selects the unit elements used in the D/A conversion sequentially, always starting with the next unused element. Ideally, this translates mismatch into high frequency noise. However, when the input to the converter is periodic, the mismatch can translate into tones in the converter baseband spectrum.

\section{Filter}

The filter consists of five stages with different clock domains, which are generated phase synchronous from one master clock, to reduce the power consumption [4], [5]. The Filter is designed in VHDL and synthesized with Synopsys Design Compiler. The structure is shown in Fig. 3.

The filter includes a 512 times decimation. It has a bandpass characteristic with cut-off-frequencies at $0.67 \mathrm{~Hz}$ and $40 \mathrm{~Hz}$, with the transfer function shown in Fig 4. As an additional feature it is possible to double the data rate and system clock for $200 \mathrm{~Hz}$ recordings, to log the electrocardiogram up to $100 \mathrm{~Hz}$. Using this mode an additional zero is added to the transfer function at $50 \mathrm{~Hz}$ to suppress power line interferences.

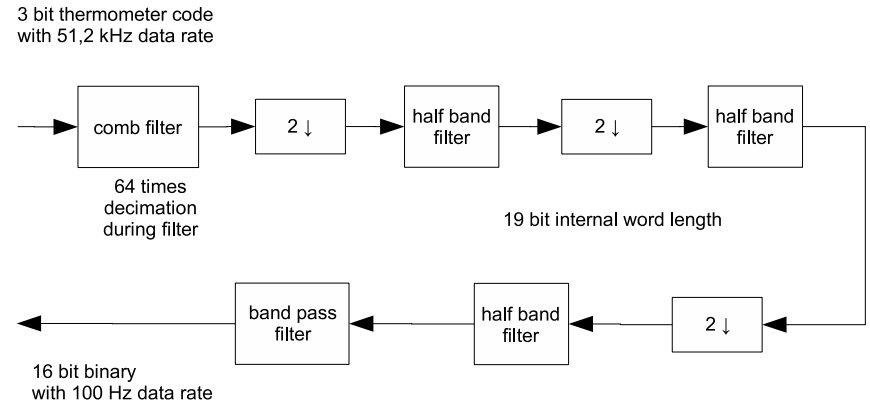

Fig. 3. Filter block diagram



Fig. 4. Pass band attenuation 


\section{I/O Interface}

A synchronous serial interface with a $256 \times 8$ bit SRAM buffer is used for the data output. The buffer can store data sets of $420 \mathrm{~ms}$ to enable a block transfer. An interrupt signal is created before transmission to signalize the next transfer and for synchronization control. The output data has an optional resolution of 8 or 16 bits with a data rate of $100 \mathrm{~Hz}$. The transfer of 42 data sets in the 16 bit mode needs only $14 \mathrm{~ms}$ which results in a duty cycle of $3.3 \%$.

\section{LAYOUT}

The ASIC was fabricated in a $0.35 \mu \mathrm{m}$ standard 2P4M CMOS process from austriamicrosystems. The chip size is $4.5 \mathrm{~mm} \mathrm{x}$ $5.5 \mathrm{~mm}$, with a total area of $24.75 \mathrm{~mm}^{2}$. A chip photograph is shown in Fig. 5.

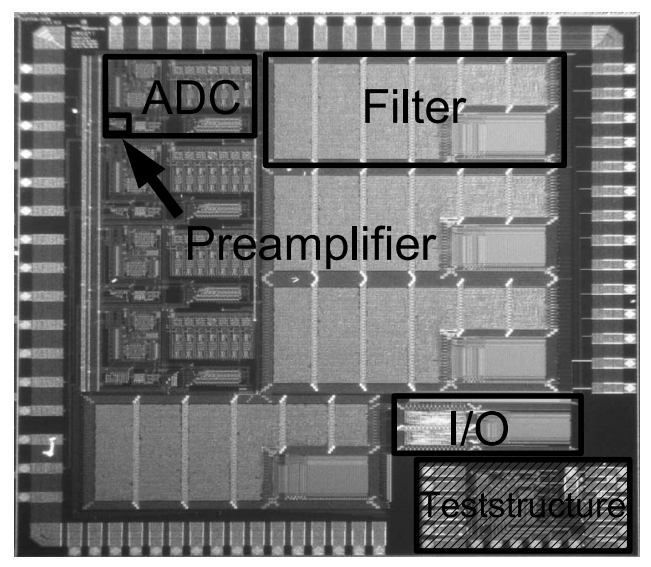

Fig. 5. Chip photograph

\section{MEASUREMENT AND DISCUSSION}

The chip is bonded in a JLCC68 package and attached to a test circuit board. All power supplies and reference voltages are connected together. The supply voltages of $-1.5 \mathrm{~V}$ and $+1.5 \mathrm{~V}$

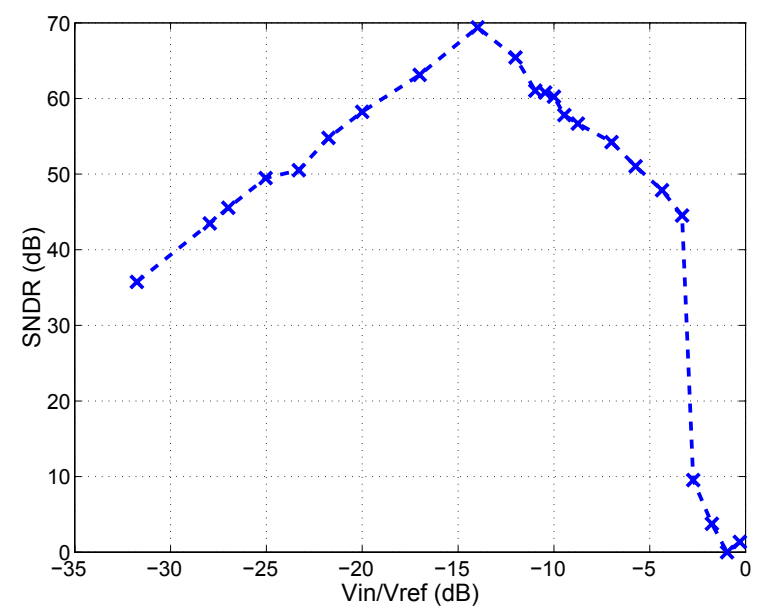

Fig. 6. SNDR at different input amplitudes are taken from two batteries. The main clock of $409.6 \mathrm{kHz}$ is fed to the board single ended with an amplitude of $3.0 \mathrm{~V}$. The recorded output data length is 1792 words. All signal processing is made by Matlab. For signal analysis in frequency domain the output data length were reduced to 1024 words.

The SNDR of different amplitudes is shown in Fig 6. A sinusoidal signal with a frequency of $10 \mathrm{~Hz}$ is used as input. The maximal SNDR is $70 \mathrm{~dB}$ with an input signal of $132 \mathrm{mV}$.

The output spectrum generated by an $10 \mathrm{~Hz}$ sinusoidal input signal with an amplitude of $50 \mu \mathrm{V}_{\mathrm{pp}}$ is shown in Fig. 7. The SNDR of this signal is $2.52 \mathrm{~dB}$ leading to an equivalent noise voltage of $13.2 \mu \mathrm{V}_{\text {rms. }}$.



Fig. 7. Power spectral density for a $50 \mu \mathrm{V}_{\mathrm{pp}}$ input signal

The system is able to measure a $6 \mathrm{mV}_{\mathrm{pp}}$ input signal with an offset voltage up to $\pm 300 \mathrm{mV}$. Measurements with different offset voltages are shown in Fig. 8.

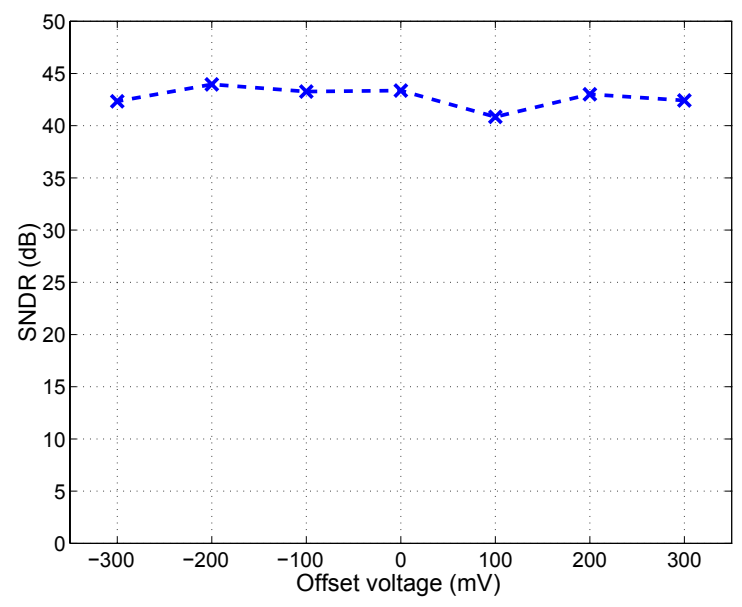

Fig. 8. SNDR of a $6 \mathrm{mV}_{\mathrm{pp}}$ signal with different offset voltages

A SNDR of $43.4 \mathrm{~dB}$ is necessary to reach a step size of $50 \mu \mathrm{V}$ with an input signal of $6 \mathrm{mV}_{\mathrm{pp}}$, as it is required by the IEC standard. The measurement results show that the SNDR is slightly below the requested value. The starcoupled resistors 
typical used in a Goldberger measurement scheme to generate a common reference voltage are integrated [7]. The small current flowing through these resistors produces a voltage drop across the ESD protection resistors inside of the pads. This voltage drop results in a reduced amplification of the input signal and a lower SNDR as estimated. The measured amplification is two.

Fig. 9 shows the pass band attenuation of the system normalized with a $10 \mathrm{~Hz}$ signal.

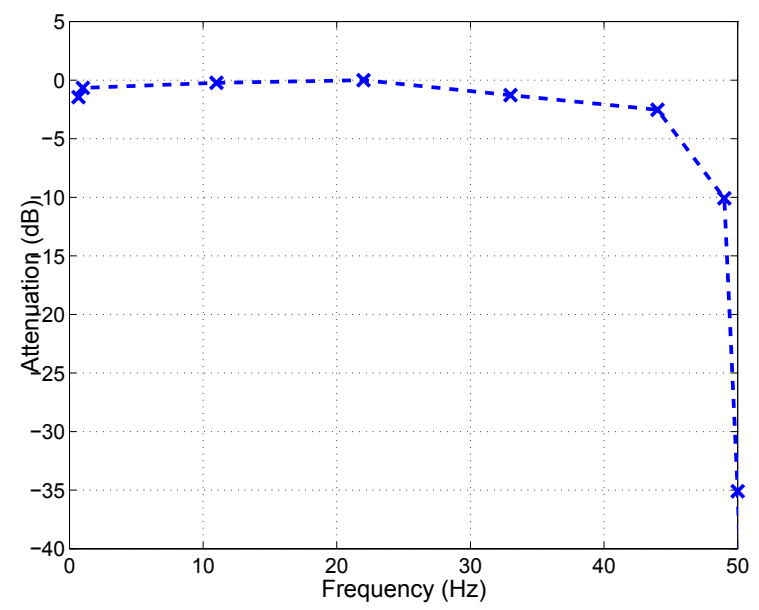

Fig. 9. Attenuation vs frequency

A recorded ECG signal is shown in Fig. 10. It was applied with a ECG simulator and shows a typical curve progression measured between the common reference and the left leg.

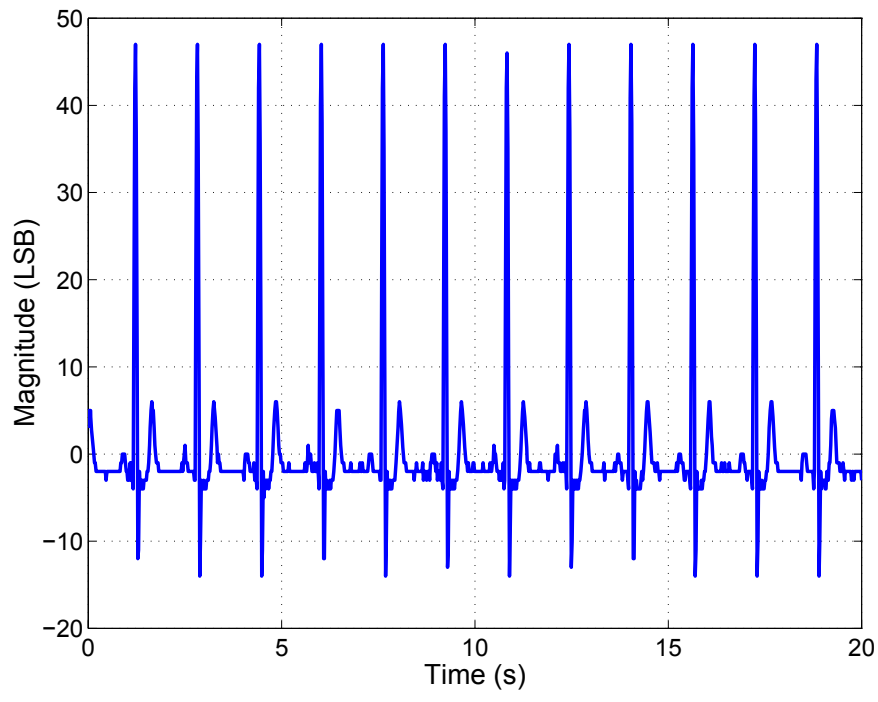

Fig. 10. Measured ECG signal
The main characteristics of the circuit are listed in Table I.

TABLE I

CIRCUIT CHARACTERISTICS

\begin{tabular}{|l|l|}
\hline input signal & $6 \mathrm{mV}$ \\
\hline DC-offset & $\pm 300 \mathrm{mV}$ \\
\hline input resistance & $>10 \mathrm{M} \Omega$ \\
\hline output data width & $8 / 16 \mathrm{bits}$ \\
\hline output data rate & $100 \mathrm{~Hz}$ \\
\hline current consumption(analog) & $441 \mu \mathrm{A}$ \\
\hline current consumption(references) & $3.4 \mu \mathrm{A}$ \\
\hline current consumption(digital) & $186 \mu \mathrm{A}$ \\
\hline total power consumption & $1.89 \mathrm{~mW}$ \\
\hline chip area & $24.75 \mathrm{~mm}^{2}$ \\
\hline
\end{tabular}

\section{CONCLUSIONS AND FURTHER WORK}

The design of an integrated ECG measuring system is presented. The implementation of several parts of the system with a $0.35 \mu \mathrm{m} 2 \mathrm{P} 4 \mathrm{M}$ CMOS process are shown and measurement results are discussed.

The requirements given by the IEC60601-2-47 standard for ambulatory ECG equipment are met, except of a step size of $50 \mu \mathrm{V}$ with an input signal of $6 \mathrm{mV}_{\mathrm{pp}}$. To solve this problem the starcoupled resistors, which generate a common reference voltage, will be merged with the feedback network of the preamplifiers in next versions of the ASIC.

The very low power consumption is the unique selling point of this integrated circuit including ADC and filter in the field of single-chip ECG measuring systems with minimal external components.

\section{REFERENCE}

[1] Particular requirements for the safety, include essential performance, of ambulatory electrocardiographic systems: International Standard IEC 60601-2-47. International Electrotechnical Commission, Geneva, Switzerland, 2001.

[2] J. Johansson, M. Völker, J. Eliasson, Å. Östmark, P. Lindgren, and J. Delsing, "MULLE: A Minimal Sensor Networking Device - Implementation and Manufacturing Challenges", Proc. IMAPS Nordic 2004, pp. 265-271, 2004.

[3] Johansson, Neubauer, Hauer: A 16-bit $\mu \mathrm{W}$ Multi-Bit $\Sigma \Delta$ Modulator for Portable ECG Applications. 29th European Solid State Conference, (2003) page. 161...164, Lisboa, Portugal.

[4] Völker, Matthias: Entwicklung eines digitalen Filters für mobile Elektrokardiographen mit sehr niedriger Leistungsaufnahme. Technische Universitaet Berlin, Nov 2004.

[5] Völker, Dorn, Johansson, Neubauer, Hauer: A digital filter for mobil ECG measurement systems. IEEE International Workshop on Medical Measurment and Applications, (2006). Benevento, Italy.

[6] Medical electrical equipment - Particular requirements for the safety of electrocardiographs: International Standard IEC 60601-2-25, International Electrotechnical Commission, Geneva, Switzerland,1999.

[7] Webster, J.G.: Medical Instrumentation, Application and Design. John Wiley, New York, 1998 